\title{
EFFECTIVE THERAPEUTIC INTERVENTIONS TO PROMOTE RECOVERY FROM FIRST PSYCHOTIC EPISODE AND MINIMIZE ITS RELAPSE: A QUALITATIVE STUDY FROM PAKISTAN
}

\author{
Rehana Khalil, Zahid Naeem*, Nazia Jameel ${ }^{* *}$, Uroosa Talib*** \\ Unaizah College of Medicine and Medical Sciences, Qassim University, Kingdom of Saudi Arabia, *Shifa Tameer-e-Millat University, Islamabad Pakistan, \\ **Baqai Medical University, Karachi Pakistan, ***Karwan-e-Hayat Psychiatric Care and Rehabiliation Center, Karachi Pakistan
}

\begin{abstract}
Objective: To explore effective therapeutic strategies to promote recovery from first episode of psychosis and minimize its relapse.

Study Design: Qualitative study.

Place and Duration of Study: Karwan-e-Hayat Psychiatric Care and Rehabilitation Center, Karachi, from Mar to Jun 2019.

Methodology: Seventeen participants were recruited through purposive sampling and data was collected through in-depth interviews. Qualitative thematic content analysis was done through generation of a coding scheme.

Results: Almost half i.e., $8(47 \%)$ had experience of 10 years, while 7 (41\%) had 15 years' experience, and $2(12 \%)$ had 30 years' experience as psychiatrists. Analysis of the interview transcripts revealed three main themes related to psychiatrists' views on interventions for first episode of psychosis to prevent relapse: (1) duration of first episode of psychosis (2) compliance and rate of recovery after first episode (3) effective interventions for first episode psychosis.

Conclusion: The effective interventions to promote recovery from first psychosis episode and minimize its relapse included multiple combinations of integrated stage-specific approaches necessitating antipsychotics (pharmacological) and psychosocial support (non-pharmacological).
\end{abstract}

Keywords: Episode, Interventions, Psychotic, Qualitative study, Recovery, Relapse, Therapeutic.

How to Cite This Article: Khalil R, Naeem Z, Jameel N, Talib U. Effective Therapeutic Interventions to Promote Recovery from First Psychotic Episode and Minimize Its Relapse: A Qualitative Study from Pakistan. Pak Armed Forces Med J 2021; 71(5): 1624-1629. doi: https://doi.org/10.51253/pafmj.v71i5.3139

This is an Open Access article distributed under the terms of the Creative Commons Attribution License (http://creativecommons.org/licenses/by/4.0), which permits unrestricted use, distribution, and reproduction in any medium, provided the original work is properly cited.

\section{INTRODUCTION}

Psychosis is listed as a feature of multiple psychiatric disorders presented in the Diagnostic and Statistical Manual of Mental Disorders-5 (DSM-5). Although psychosis is the defining feature of the schizophrenia spectrum disorders (i.e., schizophrenia, schizoaffective disorder, delusional disorder, schizophreniform disorder, and brief psychotic disorder), it also occurs in some people with bipolar disorder during either a manic or depressive episode as well as in some individuals during a major depressive episode associated with major depressive disorder. ${ }^{1}$ Psychotic symptoms (i.e., hallucinations without insight, delusions) may develop during either intoxication or withdrawal from substances and, in some cases, may become chronic sequelae of prior substance use (substance induced psychotic disorder). ${ }^{1}$ The course and outcome of first psychotic episode is greatly dependent on its initial management. ${ }^{2}$ Early intervention means early detection, shortening delays in effective treatment, and providing optimal and sustained treatment. ${ }^{3}$ The notion

Correspondence: Dr Rehana Khalil, House No. B-67, Block-13, Federal B Area, Karachi Pakistan

Received: 31 Jul 2019; revision received: 07 Sep 2020; accepted: 09 Sep 2020 that optimal treatment of the early phase of disorder could shorten the duration of illness and thus reduce the prevalence of the disorder, and further have a positive medium to long term effect on the course and outcome, is an attractive idea. The primary task of therapists involved in the management of patients who have experienced a first episode of psychosis is promotion of recovery and prevention of secondary morbidity, relapse and persistent disability. ${ }^{1}$ For the early psychosis management, the psychiatrist should keep in mind that psychosis and schizophrenia are not the same illnesses. Psychosis is the name of symptoms or experiences, which include hallucinations and delusions. ${ }^{4}$ Diagnosis in early psychosis can be highly unstable. Thus, early diagnosis of schizophrenia should be avoided. However, the treatment should be phase specific. This introduces the idea of 'staging' into psychiatric treatment, an idea which has been rarely applied. It implies that the treatment in earlier phases of illness should be different, more benign and potentially more effective than in later phases. ${ }^{1}$ The foremost goal of our exploratory study is to identify a clinically useful strategy for first episode of psychosis, which can take care of different kinds of concerns that arise along the 
continuum as people move toward recovery and minimize relapse of the psychosis.

\section{METHODOLOGY}

Qualitative approach with in-depth Interviews was adopted for this study. Data was collected from March to June 2019 at the Karwan-e-Hayat Psychiatric Care and Rehabilitation Center, Karachi, Pakistan. The study was approved by the Institutional Review Board (IRB) at KHPCRC with approval number: REF/IERB/ 102/2019. Karwan-e-Hayat maintains one of the largest numbers of psychiatric inpatients in Karachi. KHPCRC at keameri comprises of a 100-bed inpatient facility providing treatment and rehabilitation services, an outpatient clinic at Khayaban-e-Jami, and a community Psychiatry Center at Korangi. Each of three facilities has an outpatient department and rehab facilities, which provide services through a multi-disciplinary team. Combined, the three facilities demonstrate stepby-step care through quality medication, therapy, counselling, and rehabilitation.

Inclusion Criteria: Psychiatrists, clinical psychologists, occupational therapists, social workers, and rehab practitioners.

Exclusion Criteria: Non-consenting individuals were excluded.

Purposive sampling technique was adopted for the study and potential participants were contacted in their offices and were invited to participate in the study. Potential participants were given an infoma-tion sheet about purpose of the study. Those who agreed to participate were recruited for in-depth interviews.

Seventeen psychiatrists were selected through purposive sampling. The ages of $9(52.9 \%)$ of the study participants (psychiatrists) were between 55-65 years, and more than half $10(58.8 \%)$ of them were females. Almost half $8(47 \%)$ had experience of 10 years, while 7 $(41 \%)$ had 15 years experience, and 2 (12\%) had 30 years experience as psychiatrists. Although all potential participants were given a copy of the written informed consent along with contact information of the principal investigator, signed informed consent forms were not collected as these would have been the only participant identifiers.

Seventeen in-depth interviews were conducted and all participants were cognitively functional and medically stable and had work experience of more than five years. A semi-structured, open-ended interview guide was used that focused on eliciting psychiatrists' perspectives on effective strategy to manage psychosis. All participants followed the same discussion guide that allowed comparisons between various responses. Interviews lasted between 45-60 minutes. Notes were taken in local language, in order to retain the literal sense of the responses. The notes were later translated and expanded, immediately after each interview concluded. Qualitative thematic content analysis was conducted by two investigators and a preliminary coding scheme was generated. The coding scheme facilitated the systematic identification of analytic patterns that became apparent from the data, as well as theoretically important concepts. Limited demographic information was obtained from participants.

Information regarding number of patients admitted, gender distribution and daily outpatient clinics was obtained from the medical record. Data were entered and analyzed using Statistical Package for Social Sciences (SPSS) version 20. Categorical variables were summarized in the form of frequencies and percentages while quantitative statistics were summarized as mean and standard deviation. For qualitative analysis, first review was done and data was explored. Initial codes were created, followed by reviewing of those codes, revision and combining into the themes. Themes were presented in a cohesive manner

\section{RESULTS}

Demographic characteristics of the participants are shown in Table. Almost two-third 12 (70.5\%) of study participants treated $>2000$ cases throughout their professional career. Their outpatient services varied from 70-150 patients per day. Sixteen (94\%) participants reported auditory psychosis as the commonest type of psychosis.

Table: Demographic characteristics of study participants.
\begin{tabular}{l|c}
\hline Characteristics & $\mathbf{n}(\%)$ \\
\hline Age (years) & $8(47.1)$ \\
\hline $45-54$ & $9(52.9)$ \\
$55-65$ & $7(41.1)$ \\
\hline Gender & $10(58.8)$ \\
\hline Male \\
Female \\
\hline Work Experience \\
\hline 5-10 Years \\
11-15 Years & $8(47)$ \\
16-20 Years & $7(41.2)$ \\
21-15 Years & - \\
26-30 Years & - \\
\hline
\end{tabular}

Number of admitted patients in the ward were 90, out of which $45(50 \%)$ were psychotic and schizophrenic patients. Ages of 68 (75.5) admitted patients were 
between 20 to 45 years and $60(66.7 \%)$ of them were males.

Analysis of the interview transcripts revealed three main themes related to psychiatrists' views on interventions for first episode of psychosis to prevent relapse: (1) duration of first episode of psychosis (2) compliance and rate of recovery after first episode (3) effective interventions for first episode of psychosis.

\section{Theme-1: Duration of First Episode of Psychosis}

Participants had different opinions about the time duration of an episode of psychosis. Almost half i.e., $8(47 \%)$ of the participants' experience about duration of psychosis was 4-6 weeks, while $7(41 \%)$ believed that duration is highly variable among the patients and can take one year to five years to get over. A variety of reasons were offered. Some thought that it was important to know their patients' status as the following statements illustrates: "I think that it depends on the age and severity of the condition" (R1); "It usually depends on the insight and motivation of the patient" (R8);" In my view it's the type of intervention and compliance which can reduce the duration of one episode of psychosis and can prevent further worsening" (R4). One of the participants expressed it by saying "One episode of psychosis takes 1-5 years to get over completely" (R2). Another said "First episode may take years to resolve completely" (R3).

Other participants stressed that there is nothing like a time duration for a distinguished episode, " It's very difficult to say that patient has completely recovered and the episode is over but it persists in one form or another you can say it's a continuous state of anomalous experiences that cause significant distress" (R5); "Ultimately, distress is subjective-no one except for the individual experiencing distress is capable of determining what is and what is not distressing for that individual. Yes! we can say, the symptoms are managed and controlled to a good extent" (R10).

\section{Theme-2: Compliance and Rate of Recovery After First Episode}

All study participants expressed very positive attitudes towards recovery of first episode of psychosis if compliance of antipsychotic medication is good. They further elaborated the recovery in following words: "Those who have experienced some abatement of the distress and limitation caused by their subjective experiences would be recovering, and those for whom their subjective experiences no longer cause significant distress or limitation at all would be considered fully recovered" (R10). Positive prognostic factors identified by the participants included a number of factors. One participant explained, "level of insight, acceptance of illness, wish to lead a normal life, and fear of symptom deterioration are the factors which determine the prognosis of psychotic patients" (R17); "If patient is diagnosed early and gets an early treatment, plus he has no co-morbidities then there are good chances of rapid recovery" (R14); "If the cause of psychosis is substance use, like if it is substance related psychosis then the patient recovers soon after quitting the illicit drug use" (R13); "To me social and family support are the key factors for recovery" (R6); "I would say, early onset of positive symptoms, self-awareness, motivation, less delusional beliefs, less hallucinations, and regular visits to consultants can lead to recovery" (R16); "There is a triad of factors which include psychiatrist, psychologist and social support. These three factors are essential for complete recovery of the psychotic patients" (R3). All participants affirmed that treatment compliance is a fundamental requirement to accomplish the goal among psychotic patients as the following statements reflect: "The compliance of antipsychotic is very essential for recovery but unfortunately compliance is a big problem among psychotic patients" (R5); "Psychotic patients usually have treatment compliance issue. Volunteers should visit them at their homes at monthly basis" (R4); "Psychosis is a process which requires time but can be treated if medical compliance is strictly observed by a caregiver" (R9).

\section{Theme-3: Effective Interventions for First Episode Psychosis}

All participants agreed that curative treatment is not available for psychosis but symptomatic treatment through antipsychotics can be done. More than half i.e., $10(59 \%)$ participants were in favor of life-long anti-psychotics while others believed that anti-psychotics should be prescribed on-need basis. Participants identified a number of strategies which are applied on psychotic patients including anti-psychotic medication, cognitive behavioral therapy (CBT), family psycho education, regular follow-ups, rehabilitation programs and occupational therapy. Moreover, hygiene assistance, treatment adherence session, relapse prevention techniques, social support and social skill training.

When participants were asked about most effective strategy/intervention for psychosis management, there were multiple responses, which can be depicted from following statements: "Cognitive behavioral therapy (CBT) is the most reliable strategy along with antipsychotic medications in the early phase of psycho- 
sis management" (R1); "Anti-psychotics are sufficient to manage psychosis if compliance is good" (R4); "Psychosis is a treatable condition irrespective of its cause. I think family psychoeducation on side by side antipsychotic treatment is essential" (R7); "A holistic phase specific approach is helpful which includes antipsychotics, family and social support though regular motivational education according to the phase of psychosis" (R8); "The starting dose should be very low and should be increased to a target dose and held there for the effect to be evaluated. Once the patient's condition is relatively stable, home-based care is less stressful for the patient. Then the period following the recovery of first episode of psychosis is very critical and mind over mood strategy is helpful to prevent remission"(R16); "Anti-psychotics plus social training, are the two key interventions to address the psychotic episode" (R12); "I would recommend anti-psychotics plus family psychoeducation plus rehabilitation" (R14); "Families need to be involved in post crisis time to prevent future relapse" (R11); "With antipsychotic treatment and psychotherapy sessions, family support is nearly equally important and plays a great role in patient's recovery. On the other hand, lack of family support, increases the liability to develop another disorder in addition to psychosis like depression or mania which can increase the severity of the psychosis" (R10); "Rehabilitation is necessary to prevent relapse of psychosis but most of the patients could not get full rehabilitation due to economic constraints and distance from the clinic" (R2); "Psychotic patients usually show childish behavior and anxiety. So, day care involvement for long-term sustainability is a good idea" (R10).

\section{DISCUSSION}

According to DSM-5's criteria, the psychotic experiences that lasts for a minimum of one month or a series of psychotic episodes the total of which is at least a month, can be termed as long-term psychosis ${ }^{5}$. The participants of our study had different opinions about the time duration of first episode of psychosis which varied from weeks to years, which may be due the fact that up to $85-90 \%$ of first episode patients achieve a remission or partial remission of their positive psychotic symptoms within the 12 months following entry to treatment, though some potentially responsive patients will fail to engage with treatment or rapidly cease adherence to medication. This is balanced by the persistence of the vulnerability in most patients and the tendency to recurrence, which may be subtle $^{6,7}$, and feels like a continuous state of psychosis.
Term non-adherence to medication is also known as non compliance of medicines. Various reasons have been cited in literature for noncompliance. These include poor insight, side effects of medicines, poor remission of symptoms, and poor therapeutic alliance. ${ }^{8}$ It also includes poor family support, ${ }^{9}$ and ignorance about need to continue treatment. ${ }^{10}$ Out study findings concurred with findings of Moritz et al ${ }^{10}$ and Baloush et $a l, 11$ that good social support and positive attitude of family members were significantly associated (74.6\%) with high compliance, ${ }^{10}$ and unfavorable attitude with noncompliance. ${ }^{11-13}$ The influence of family on compliance is a modifiable factor which can be achieved through psychoeducation. ${ }^{9}$ In addition to family-related domain, our study stress upon significance of illnessrelated domains like level of insight, acceptance of illness, self-awareness, motivation, wish to lead a normal life, fear of symptom deterioration less delusional beliefs, less hallucinations substance related causes of psychosis, and early onset of positive symptoms. These illness-related domains were associated with compliance as has been reported in earlier studies by Moritz et al, ${ }^{10}$ Baloush et al ${ }^{11}$ and Stentzel et al. ${ }^{13}$

Slade et al,14 and Frost et al, ${ }^{15}$ discovered that recovery is emerging as much more common than previously understood. Participants of this study also showed a positive attitude towards recovery from mental illnesses. Likewise, a long-term ( $\geq 20$ year) follow-up study by Slade et al ${ }^{16}$ that more than half of people given a diagnosis of schizophrenia experience clinical recovery.

Research studies by Malla et $a l^{3}$ and Gitlin et $a l^{6}$ have focused on the treated course of early psychosis. They have shown that the early course of illness for psychosis is turbulent and relapse prone, with up to $80 \%$ of patients relapsing within a five-year period, and between acute relapses there may be additional persistence of subclinical, yet disabling clinical features. These findings suggested that, if possible, drug therapy should be continued for most if not all patients same is obvious from our study which recommend continuation of antipsychotic treatment whether lifelong or on-need basis.

In line with earlier research done by Kapur et al, ${ }^{17}$ and Wunderink et al,18 a very low starting dose was suggested in early stage psychosis by our participants and then gradually increased and held there for the effect to be evaluated. Further increases should only occur in the setting of poor response and only then at intervals of approximately 3 weeks, to allow the effect 
of the change in dose to become clear. More rapid increases in dose in first episode psychosis lead to greater risk of side effects, especially extrapyramidal features, with no clear benefit. ${ }^{17}$ To meet the needs of an individual, it is important to formulate appropriate strategies for the different stages of the illness (prodromal phase, acute phase, early recovery phase and late recovery phase) because patients have different therapeutic needs at each stage. In the acute treatment, prescribing principle for first-episode psychosis are to maximize benefit and minimize side effects because the first experience of medication may influence a patient's future attitudes of therapy of all types. ${ }^{2}$

Our findings showed that non-pharmacological psychosocial interventions are also essential elements of the treatment for patients with psychosis, especially during the stable phase of illness, ${ }^{19}$ and a range of psychosocial strategies can augment and broaden the scope and depth of the recovery process, ${ }^{20}$ and these interventions include patient and family education, cognitive behaviorally oriented therapies, family interventions, assertive community treatment, social skills training, and supported employment. Caregiver and family support also should be provided concurrently with patient-oriented therapies. ${ }^{20}$

Our study participants were of a unanimous opinion that psychosis can be addressed through integrated approach of various psychosocial strategies in addition to the optimal antipsychotic medication, and our findings about effective treatment interventions were in agreement with research done by Falloon et al, ${ }^{21}$ Wang et $a l^{22}$ and Kidd et al, ${ }^{23}$ including antipsychotic medication, CBT sessions which focused on thought modification, Fami-ly psychoeducation, regular follow-ups, rehabilita-tion programs like day care activities (i.e., leisure life activities and career-based stress management training for skill building) and occupational therapy. Moreover, hygiene assistance, treatment adherence session, relapse prevention techniques, social support and social skill training.

\section{RECOMMENDATION}

Our research has identified the practical strategies for psychosis management in a developing country. There is a need to introduce peer support which simply means receiving support directly from others who have "faced the same condition and recovered." Peer support can be done through peer-run crisis homes, 24-hour crisis hotlines, support groups and classes. It can be used either as an adjunct to any of the above methods, or even stand entirely alone as the primary source of support in areas with a strong peer support network.

\section{Implications for Future Research}

The dominant paradigm for psychosis in the mental health field today is brain-disease model ${ }^{1}$. It's important to look closely at the validity of this model because if psychosis is a brain disease then it should have a biological cure like an infection but in reality, but in reality mere antipsychotics are ineffective and need augmentation of psychosocial construct to become effective.

Vast majority of psychosis research has paid almost no attention to the subjective experiences of the patients. It's time to shift our attention to the actual lived experiences of the individuals to fully understand the most fundamental levels of experience during psychosis and exploring what factors are most influential in each stage. This will help the researchers to formulate an integrative and comprehensive model of psychosis.

\section{CONCLUSION}

An integrated stage-specific approach including antipsychotics (pharmacological) and psychosocial support (nonpharmacological) is likely to prove beneficial in promoting complete recovery from first episode of psychosis irrespective of its cause and minimize its relapse. Integrated stagespecific approach is a strategy which includes a balanced combination of required dose of anti-psychotic medication with case-specific proportion and duration of cognitive behavioral therapy, Family Psychoeducation, Regular followups, Rehabilitation programs and Occupational therapy.

\section{Conflict of Interest: None.}

\section{Authors' Contribution}

RK: Conceived designed \& analysis, ZN: Data Analysis, review \& editing, NJ: Data collectin, interpretation \& manuscript writing, UT: Data collectin, interpretation \& manuscript writing.

\section{REFERENCES}

1. Arciniegas DB. Psychosis. Continuum (Minneap Minn) Behavioral Neurol Neuropsych 2015; 21(3): 715-736.

2. Douki S, Taktak MJ, Ben Zineb S, Cheour M. Therapeutic strategies in the first psychotic episode. Encephale 1999; 25(3): 44-51.

3. Malla AK. Norman RMG. Early intervention in schizophrenia and related disorders: advantages and pitfalls. Curr Opin Psy 2002; 15(1): 17-23.

4. Rethink mental illness. Advice and information about mental illness/learn more about conditions cannabis and mental health. [Internet]. Available from: https://www.rethink.org/adviceand-information/about-mental-illness/learn-more-aboutconditions/ cannabis-and-mental-health/ (Accessed on July 18, 2019). 


\section{First Psychotic Episode}

5. Norman RMG, Malla AK. Duration of untreated psychosis: a critical examination of the concept and its importance. Psychol Med 2001; 31(3): 381-400.

6. Gitlin M, Nuechterlein K, Subotnik KL, Ventura J, Mintz J, Fogelson $\mathrm{DL}$, et al. Clinical out- come following neuroleptic discontinuation in patients with remitted recent-onset schizophrenia. Am J Psychiatry 2001; 158(11): 1835-1842.

7. Thompson A, Winsper C, Marwaha S, Haynes J, Alvarez-Jimenez $M$, Hetrick S, et al . Maintenance antipsychotic treatment versus discontinuation strategies following remission from first episode psychosis: systematic review. BJ Psych Open 2018; 4(4): 215-225.

8. Moritz S, Favrod J, Andreou C, Morrison AP, Bohn F, Veckenstedt $\mathrm{R}$, et al. Beyond the usual suspects: positive attitudes towards positive symptoms is associated with medication non compliance in psychosis. Schizophr Bull 2013; 39(4): 917-922.

9. Razali MS, Yahya H. Compliance with treatment in schizophrenia: a drug intervention program in a developing country. Acta Psychiatr Scand 1995; 91(5): 331-335.

10. Moritz S, Hünsche A, Lincoln TM. Nonadherence to antipsychotics: the role of positive attitudes towards positive symptoms. Eur Neurop Pharmacol 2014; 24(11): 1745-1752.

11. Baloush-Kleinman V, Levine SZ, Roe D, Shnitt D, Weizman A, et al. Adherence to antipsychotic drug treatment in early-episode schizophrenia: a six-month naturalistic follow-up study. Schizophr Res 2011; 130(1-3): 176-181.

12. Rao KN, George J, Sudarshan CY, Begum S. Treatment compliance and noncompliance in psychoses. Ind J Psych 2017; 59(1): 69-76.

13. Stentzel U, van den Berg N, Schulze LN, Schwaneberg T, Radicke F, Langosch JM, et al. Predictors of medication adherence among patients with severe psychiatric disorders: findings from the baseline assessment of a randomized controlled trial (Tecla). BMC Psych 2018; 18(1): 155-158.
14. Slade M, Amering M, Farkas M, Hamilton B, O'Hagan M, Panther $\mathrm{G}$, et al. Uses and abuses of recovery: implementing recovery-oriented practices in mental health systems. World Psych 2014; 13(1): 12-20.

15. Frost BG, Tirupati S, Johnston S, Turrell M, Lewin TJ, Sly KA, et al. An Integrated recovery-oriented model (IRM) for mental health services: evolution and challenges. BMC Psych 2017; 17(1): 22-28.

16. Slade M, Amering M, Oades L. Recovery: an international perspective. Epidemiol Psichiatr Soc 2008; 17(2): 128-137.

17. Kapur S, Zipursky R, Jones C. Relationship between dopamine D2 occupancy, clinical response, and side effects: a double-blind PET study of first-episode schizophrenia. Am J Psych 2000; 157(4): 514-520.

18. Wunderink L. Personalizing antipsychotic treatment: evidence and thoughts on individualized tailoring of antipsychotic dosage in the treatment of psychotic disorders. Ther Adv Psychopharmacol 2019; 8(9): 2045125319836566.

19. Lehman AF, Lieberman JA, Dixon LB. Practice guideline for the treatment of patients with schizophrenia, $2^{\text {nd }}$ ed. Arlington: Am J Psych 2004; 161(Suppl-2): 1-56.

20. Malla AK, Norman RMG. Early intervention in schizo- phrenia and related disorders: advantages and pitfalls. Curr Opin Psych 2002; 5(1): 17-23.

21. Falloon IR, Held T, Roncone R. Optimal Treatment Strategies to Enhance Recovery from Schizophrenia. Aust. NZJ Psychol 1998; 32(1): 43-49.

22. Wang W, Zhou Y, Chai N, Liu D. Cognitive-behavioural therapy for personal recovery of patients with schizophrenia: A systematic review and meta-analysis. Gen Psych 2019; 32(4): e100040.

23. Kidd S, Kenny A, McKinstry C. The meaning of recovery in a regional mental health service: an action research study. J Adv Nurs 2015; 71(1): 181-192. 